\title{
Risk Factors for Cement Loosening after Vertebroplasty for Osteoporotic Vertebral Fracture with Intravertebral Cleft: A Retrospective Analysis
}

\author{
Toshio Nakamae ${ }^{1}$, Kiyotaka Yamada ${ }^{1}$, Yasuyuki Tsuchida ${ }^{2}$, \\ Orso Lorenzo Osti ${ }^{3}$, Nobuo Adachi ${ }^{4}$, Yoshinori Fujimoto ${ }^{1}$ \\ ${ }^{I}$ Department of Orthopaedic Surgery, JA Hiroshima General Hospital, Hiroshima, Japan \\ ${ }^{2}$ Department of Radiology, JA Hiroshima General Hospital, Hiroshima, Japan \\ ${ }^{3}$ Spinal Service, Calvary Health Care, North Adelaide Campus, North Adelaide, SA, Australia \\ ${ }^{4}$ Department of Orthopaedic Surgery, Integrated Health Sciences, Institute of Biomedical and Health Sciences, Hiroshima University, Hiroshima, Japan
}

Study Design: Retrospective case-control study.

Purpose: To evaluate the primary outcomes and radiographic results of percutaneous vertebroplasty (PVP) in patients with singlelevel osteoporotic vertebral fracture (OVF) with intravertebral cleft (IVC) to identify the risk factors for cement loosening after PVP.

Overview of Literature: PVP is a widely accepted method for managing painful OVF; however, cement loosening occasionally occurs with poor outcomes.

Methods: This retrospective study involved 195 patients treated with PVP for single-level OVF with IVC. Six months thereafter, the primary outcomes were evaluated using the Visual Analog Scale (VAS) for back pain and the modified Oswestry Disability Index. Computed tomography was conducted to detect cement loosening. Possible risk factors, such as age, sex, wedging angle, intravertebral instability, Parkinson's disease, spinous process fracture, ankylosing spinal hyperostosis, split vertebrae, and adjacent intervertebral vacuum, were assessed.

Results: Forty-nine patients (25\%) experienced cement loosening 6 months after PVP. The mean VAS scores were significantly higher in patients with cement loosening than in those without (50 vs. $26 \mathrm{~mm}$, respectively; $p<0.01$ ). Cement loosening was closely associated with intravertebral instability (odds ratio [OR], 1.20; 95\% confidence interval [CI], 1.04-1.40; $p=0.015)$, Parkinson's disease (OR, $54.31 ; 95 \% \mathrm{Cl}, 4.47-659.53 ; p=0.002)$, spinous process fracture (OR, 7.11; $95 \% \mathrm{Cl}, 1.65-30.60 ; p=0.009)$, and split vertebrae (OR, $11.59 ; 95 \% \mathrm{Cl}, 1.64-82.02 ; p=0.014)$.

Conclusions: Patients with cement loosening experienced worse back pain than those without cement loosening. The important risk factors that influenced cement loosening after PVP were high intravertebral instability, Parkinson's disease, spinous process fracture, and split vertebrae.

Keywords: Percutaneous vertebroplasty; Osteoporotic vertebral fracture; Cement loosening; Intravertebral cleft; Spinous process fracture

Received Dec 21, 2017; Revised Jan 18, 2018; Accepted Mar 4, 2018

Corresponding author: Toshio Nakamae

Department of Orthopaedic Surgery, JA Hiroshima General Hospital, Hiroshima, Japan, 1-3-3 Jigozen, Hatsukaichi, Hiroshima, 7388503, Japan

Tel: +81-829-36-3111, Fax: +81-829-36-5573, E-mail: toshinakamae623813@yahoo.co.jp 


\section{Introduction}

Osteoporotic vertebral fractures (OVFs) are becoming increasingly common in medical practice owing to the aging of the population. OVFs usually heal without severe pain or neurologic complications. However, rigorous follow-up studies have shown that some OVFs can lead to severe deterioration in health and quality of life owing to persistent debilitating back pain and reduced activity [1]. In particular, patients with OVF with an intravertebral cleft (IVC) experience chronic back pain [2-4]. Intravertebral instability caused by IVC is clinically significant in painful cases of OVF [5].

Percutaneous vertebroplasty (PVP) is a minimally invasive and generally effective method of vertebral augmentation for treating painful OVF with IVC [6-10]. Early clinical improvement has been observed following this procedure in more than $80 \%$ of the patients [11]. However, some patients experience cement loosening with recurrence of back pain after vertebral augmentation [1214]. An accurate understanding of the factors leading to cement loosening after PVP would help surgeons and patients make well-informed decisions about whether to perform the procedure and would also enable more accurate predictions of outcomes. However, few studies have reported factors that may influence the results of PVP [15-17]. Here, we aimed to evaluate the primary outcomes and radiographic results of PVP for patients with singlelevel OVF with IVC between patients with and without cement loosening to identify the associated risk factors.

\section{Materials and Methods}

\section{Patient selection}

Between 2003 and 2010, we investigated patients with single-level OVF with IVC detected using preoperative radiography, computed tomography $(\mathrm{CT})$, and magnetic resonance imaging (MRI) at JA Hiroshima General Hospital. The selection criteria for PVP were as follows: (1) minimum level of back pain (Visual Analog Scale [VAS]; range, $0-100 \mathrm{~mm}) \geq 40 \mathrm{~mm}$, refractory to standard medical treatment, comprising bed rest, analgesic administration, and/or external back bracing for $\geq 3$ months and (2) radiographic evidence of single-level OVF with IVC consistent with the spinal level of local tenderness. Patients with spinal cancer, active infection, and/or uncorrectable bleeding disorders; those who did not provide informed consent; and those unlikely to comply with direct followup were excluded. This study was approved by the institutional review board of JA Hiroshima General Hospital (approval no., E-1098), and prior to study participation, all patients provided written informed consent to undergo PVP.

\section{Percutaneous vertebroplasty procedure}

All PVPs were performed by experienced spine surgeons using biplane fluoroscopic guidance under general anesthesia. Two 14G bone needles were inserted into the IVC using the bilateral transpedicular approach. Before injecting bone cement, the capacity of the IVC was measured using contrast medium after performing a "cavitygram." Residual contrast medium was washed out with normal saline to adequately clear the IVC. By injecting the same volume of polymethylmethacrylate bone cement as that of the IVC, the injection was safely administered using a one-sided needle without exerting additional pressure $[9,10]$.

\section{Clinical and radiological assessments}

Six months after PVP, back pain and low back-specific physical function were assessed as per the VAS score and the American Academy of Orthopedic Surgeons MODEMS version of the Oswestry Disability Index (ODI; range, $0 \%-100 \%)[18,19]$. Cement loosening was identified as a low-density area between the vertebral body and the cement on CT at 6 months after PVP (there was no evidence of cement loosening immediately after PVP) (Fig. $1 \mathrm{~A}, \mathrm{~B})$.

Risk factors that could possibly affect cement loosening were retrospectively reviewed, and were as follows: (1) clinical parameters: age, sex, and Parkinson's disease and (2) radiological parameters: wedging angle of the fractured vertebrae (measured as the angle between cephalic upper and lower endplates on a lateral radiogram with the patient in the sitting position) (Fig. 1C), intravertebral instability of the affected vertebra (measured as the difference between wedging angle on lateral radiograms with the patient in the sitting and supine positions) (Fig. 1C, D), spinous process fracture (Fig. 1E), ankylosing spinal hyperostosis, split vertebrae (Fig. 1F), and adjacent intervertebral vacuum. 

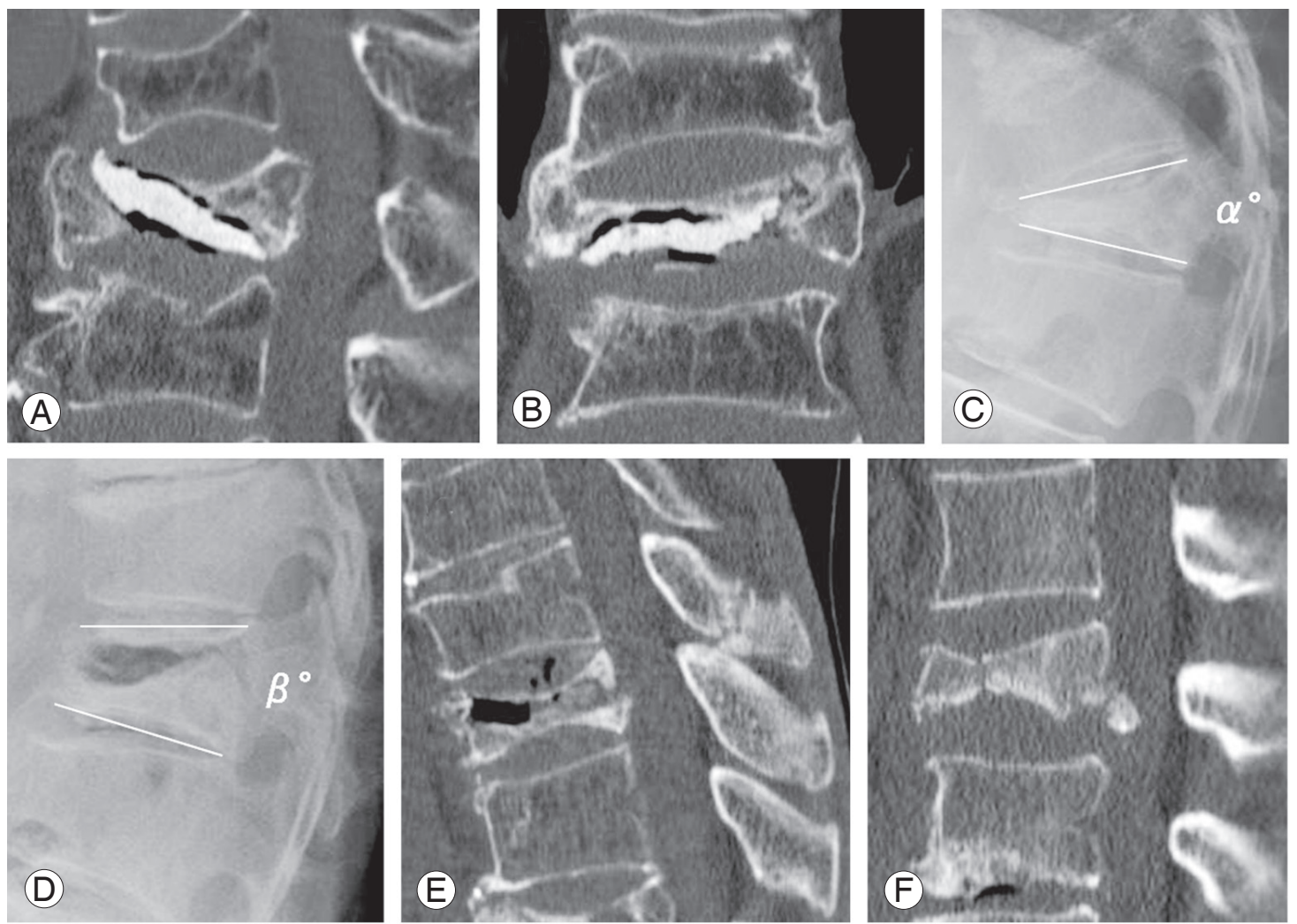

Fig. 1. Radiological assessments. Cement loosening after vertebroplasty for osteoporotic vertebral fracture with intravertebral cleft. Cement loosening was defined as a low-intensity area between the vertebral body and the bone cement on CT 6 months after percutaneous vertebroplasty (A: sagittal, B: coronal). Wedging angle of the fractured vertebrae measured as the angle between cephalic upper and lower endplates on a lateral radiogram with the patient in the sitting position (C). Intravertebral instability of the affected vertebra measured as the difference between the wedging angle on lateral radiograms with the patient in the sitting and supine positions (C, D: $\alpha-\beta)$. Spinous process fracture (E: sagittal, CT). Split vertebrae (F: sagittal, CT). CT, computed tomography.

The patients were followed-up directly and periodically after PVP. Orthopedic surgeons not involved in the treatment performed the follow-up and clinical examinations to assess the neurologic recovery and functional status. The VAS and ODI questionnaires were self-administered to avoid interviewer bias.

\section{Statistical analyses}

Using the Wilcoxon signed-rank test, Mann-Whitney $U$ test, and/or Bonferroni-Dunn post-hoc test, clinical and radiographic parameters between patients with or without cement loosening were compared to analyze the risk factors that cause cement loosening after PVP.

Associations between cement loosening and preoperative parameters were analyzed using multivariate logisticregression analyses. Statistical significance was defined at $p<0.05$ for a two-sided hypothesis. Mean values are presented as \pm standard deviation values. All data analyses were performed using SPSS ver. 16.0 (SPSS Inc., Chicago, IL, USA).

\section{Results}

\section{Baseline characteristics of the patients}

Total 195 patients (50 men, 145 women) with single OVF with IVC were enrolled. Mean age at diagnosis was 77 years (range, 57-93 years). Mean time-period from the onset of acute fracture to the PVP procedure was 38 weeks (range, 12-144 weeks). OVF with IVC was detected from the T8 to L5 level and occurred at the thoracolumbar junction in $80 \%$ of the patients. The mean VAS and ODI scores were $87 \pm 14 \mathrm{~mm}$ and $59.1 \% \pm 7.2 \%$ before PVP and 
$31 \pm 3 \mathrm{~mm}$ and $37.0 \% \pm 5.1 \%$ at 6 months after PVP, respectively (Fig. 2).

\section{Assessment of preoperative parameters}

Of the 195 patients with OVF, 41 (20.5\%) had spinous process fracture, 13 (6.7\%) had Parkinson's disease, 21 (10.8\%) had a split vertebra, 18 (9.2\%) had ankylosing spinal hyperostosis, and 29 (14.9\%) had an adjacent intervertebral vacuum. Forty-nine patients (25\%) exhib- ited cement loosening 6 months after PVP. Five patients underwent additional instrumented surgery because of pain and/or motor involvement. Six months after PVP, the mean VAS in the patients with and without cement loosening was $50 \pm 25 \mathrm{~mm}$ and $26 \pm 27 \mathrm{~mm}$, respectively (Table 1). Thus, the mean VAS was significantly higher in the patients with cement loosening than in those without $(p<0.01)$.
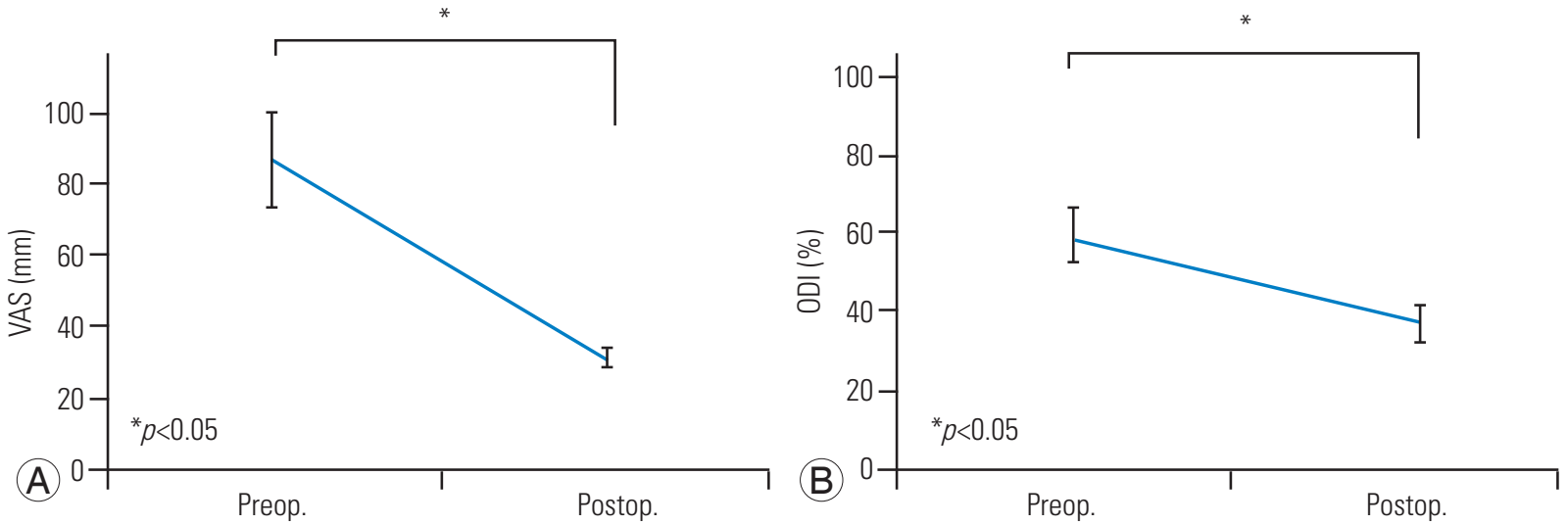

Fig. 2. Changes in the VAS (A) and ODI (B) scores. Total 195 patients with single osteoporotic vertebral fracture with intravertebral cleft were analyzed. The mean VAS and modified ODI scores were significantly lower at 6 months after PVP than those before PVP (A: VAS, B: ODI). VAS, Visual Analog Scale; ODI, Oswestry Disability Index; PVP, percutaneous vertebroplasty; Preop., preoperative; Postop., postoperative.

Table 1. Mean Visual Analog Scale scores in patients with and without cement loosening

\begin{tabular}{lccc} 
& Loosening $(+)$ & Loosening $(-)$ & $p$-value \\
Visual Analog Scale $(\mathrm{mm})$ & $50 \pm 25$ & $26 \pm 27$ & $<0.01$ \\
\hline
\end{tabular}

Values are presented as mean \pm standard deviation.

Table 2. Preoperative parameters in patients with and without cement loosening

\begin{tabular}{lccc} 
Preoperative parameters & Loosening $(+)$ & Loosening $(-)$ & $p$-value \\
Mean age $(y r)$ & 76.8 & 77.3 & 0.240 \\
Women & $39(73.3)$ & 25.9 & 0.672 \\
\hline Wedging angle $\left(^{\circ}\right)$ & 36.3 & 9.8 & 0.980 \\
\hline Intravertebral instability $\left(^{\circ}\right)$ & 6.6 & $11(28.9)$ & $<0.05$ \\
\hline Parkinson's disease & $2(1.9)$ & $20(40.8)$ & $<0.01$ \\
\hline Spinous process fracture & $21(14.4)$ & $6(12.2)$ & $<0.01$ \\
\hline Ankylosing spinal hyperostosis & $12(8.2)$ & $16(32.7)$ & 0.337 \\
\hline Split vertebrae & $5(3.4)$ & $14(28.6)$ & $<0.01$ \\
\hline Adjacent intervertebral vacuum & $15(10.3)$ & $<0.05$ \\
\hline
\end{tabular}

Values are presented as number (\%). 
Table 3. Factors related to cement loosening

\begin{tabular}{lcc} 
Independent variable & Odds ratio (95\% confidence interval) & $p$-value \\
Intravertebral instability & $1.20(1.04-1.40)$ & $<0.05$ \\
Parkinson's disease & $54.31(4.47-659.53)$ & $<0.01$ \\
\hline Spinous process fracture & $7.11(1.65-30.60)$ & $<0.01$ \\
Ankylosing spinal hyperostosis & $3.84(0.48-30.76)$ & 0.205 \\
Split vertebrae & $11.59(1.64-82.02)$ & $<0.05$ \\
Adjacent intervertebral vacuum & $1.54(0.30-7.96)$ & 0.606 \\
\hline
\end{tabular}

Dependent variable: cement loosening; independent variables: intravertebral instability Parkinson's disease, spinous process fracture, ankylosing spinal hyperostosis, split vertebrae, and adjacent intervertebral vacuum.

\section{Analyses of the correlation between cement loosen- ing and preoperative parameters}

Intravertebral instability was significantly higher $(p<0.05)$ in patients with cement loosening, whereas Parkinson's disease $(p<0.01)$, spinous process fracture $(p<0.01)$, split vertebrae $(p<0.01)$, and adjacent intervertebral vacuum $(p<0.05)$ were significantly more common in the nonloosening group (Table 2). Multivariate logistic-regression analyses revealed significant association between cement loosening and Parkinson's disease (odds ratio [OR], 54.31; 95\% confidence interval [CI], 4.47-659.53; $p<0.01)$, split vertebrae (OR, 11.59; 95\% CI, 1.64-82.02; $p<0.05$ ), spinous process fracture (OR, 7.11; 95\% CI, 1.65-30.60; $p<0.01$ ), and intravertebral instability (OR, $1.20 ; 95 \% \mathrm{CI}$, $1.04-1.40 ; p<0.05$ ) (Table 3 ).

\section{Discussion}

We investigated the clinical and radiographic outcomes of PVP for single-level OVF with IVC. Patients with cement loosening after PVP experienced worse back pain than those without cement loosening did. Our results indicated that cement loosening was associated with preoperative intravertebral instability, Parkinson's disease, spinous process fracture, and split vertebrae.

OVF is becoming an increasingly significant global socio-economic problem owing to the rapid growth of population. The presence of IVC reportedly leads to prolonged back pain [2-4]. PVP is reportedly useful for pain relief in the treatment of OVF with IVC [6-10]. However, few studies have assessed the factors that may influence the outcomes of PVP [15-17,20,21]. Extensive anterior vertebral height restoration, solid lump filling cement, multiple symptomatic vertebrae, American Society of Anesthesiologists status 1, presence of signal changes on MRI, vertebral body collapse to $<70 \%$, and small IVC have been suggested as factors that may lead to poor outcomes $[13,15,20,21]$. However, the precise factors that influence the outcomes after vertebroplasty for OVF remain controversial. Our results indicated that high intravertebral instability, Parkinson's disease, spinous process fracture, and split vertebrae are the risk factors for predicting intravertebral cement loosening after PVP for OVF with IVC.

Some previous studies have reported that greater height restoration is a risk factor for re-fracture of the cemented vertebrae [20,21]. Lin et al. [21] reported that re-fracture of the cemented vertebrae after vertebroplasty occurred in $63 \%$ of all osteoporotic patients and that significant anterior vertebral height restoration increased the risk of subsequent fractures in the cemented vertebrae. Vertebroplasty may lead to load shift to the anterior, thus causing re-fracture of the cemented vertebrae. In the PVP procedure, the decision regarding the restoration of the affected vertebrae is made when the patient is in the prone position during surgery. Consequently, vertebral height restoration is strongly correlated with preoperative intravertebral instability of the fractured vertebrae. Intravertebral instability reportedly reflects clinical symptoms $[3,4]$. In our study, large intravertebral instability was one of the risk factors for intravertebral cement loosening after PVP. Therefore, it is important to precisely evaluate preoperative intravertebral instability before PVP.

Genever et al. [22] reported that the risk of bone fracture was considerably high in patients with Parkinson's disease than in those with other medical conditions. When a spinal fracture occurs in patients with Parkinson's disease, surgical treatment is challenging because such pa- 
tients have poor bone quality and several comorbidities, which lead to a high complication rate [23-25]. The surgical procedure for OVF in patients with Parkinson's disease needs rigid long fusion with instrumentation [26-28]. Babat et al. [23] reported the requirement of revision surgery in 12 of the 14 patients with spinal surgery complicated by Parkinson's disease and concluded that the increased risk is attributable to persistent kyphosis or instability at the operated or adjacent vertebral levels. Few studies have examined PVP for treating vertebral fractures associated with Parkinson's disease. Our data indicated that patients with OVF and Parkinson's disease tend to experience intravertebral cement loosening after PVP, thereby warranting careful postoperative management.

Generally, vertebral fractures associated with the involvement of posterior elements, such as a spinous process fracture, are more unstable, and may also require surgical instrumentation [29]. To our knowledge, to date, only one study has assessed the presence of spinous process fractures in elderly patients with osteoporotic vertebral body fractures using MRI or CT and reported a prevalence of $3.5 \%$ in these patients [30]. In the current study, 20.5\% of the patients had spinous process fractures. This difference in prevalence may be attributable to the fact that the former study included patients with acute vertebral fractures, and majority of these patients heal without ongoing symptoms. Contrarily, our study included patients with painful chronic vertebral pseudoarthrosis. It is possible that the nonunion of the vertebral body fracture may lead to an insufficiency-type fracture of the spinous process. Another possibility is that OVF with spinous process fractures may lead to pseudoarthrosis. Here, spinous process fracture was one of the main risk factors for cement loosening after PVP. OVF patients with spinous process fractures may show greater instability, and PVP alone could not maintain the posterior column stability. Therefore, although the presence of spinous process fractures in OVF has not received much attention thus far, our findings indicate that it may represent a prognostically important factor that should be considered during treatment planning.

There are certain limitations to the present study. First, not all patients with cement loosening may experience pain and dysfunction; in fact, some patients exhibited cement loosening without any pain. Second, there is a lack of evidence-based longitudinal studies and long-term follow-up data. Third, we did not evaluate factors other than age, sex, wedging angle, intravertebral instability, Parkinson's disease, spinous process fracture, ankylosing spinal hyperostosis, split vertebrae, and adjacent intervertebral vacuum.

\section{Conclusions}

The findings of this study indicate that high preoperative intravertebral instability, Parkinson's disease, spinous process fracture, and split vertebrae are associated with intravertebral cement loosening after PVP for OVF with IVC. Moreover, patients with cement loosening had worse back pain than those without cement loosening did.

\section{Conflict of Interest}

No potential conflict of interest relevant to this article was reported.

\section{Acknowledgments}

We thank Maki Miyaki, DVM, PhD, for her helpful assistance with the statistical analysis.

\section{Author Contributions}

Toshio Nakamae wrote and prepared the manuscript, and all of the authors participated in the study design. All authors have read, reviewed, and approved the article.

\section{References}

1. Suzuki N, Ogikubo O, Hansson T. The course of the acute vertebral body fragility fracture: its effect on pain, disability and quality of life during 12 months. Eur Spine J 2008;17:1380-90.

2. Lane JI, Maus TP, Wald JT, Thielen KR, Bobra S, Luetmer PH. Intravertebral clefts opacified during vertebroplasty: pathogenesis, technical implications, and prognostic significance. AJNR Am J Neuroradiol 2002;23:1642-6.

3. Kawaguchi S, Horigome K, Yajima H, et al. Symptomatic relevance of intravertebral cleft in patients with osteoporotic vertebral fracture. J Neurosurg Spine 2010;13:267-75.

4. Nakamae T, Fujimoto Y, Yamada K, et al. Relationship between clinical symptoms of osteoporotic 
vertebral fracture with intravertebral cleft and radiographic findings. J Orthop Sci 2017;22:201-6.

5. McKiernan F, Jensen R, Faciszewski T. The dynamic mobility of vertebral compression fractures. J Bone Miner Res 2003;18:24-9.

6. Jang JS, Kim DY, Lee SH. Efficacy of percutaneous vertebroplasty in the treatment of intravertebral pseudarthrosis associated with noninfected avascular necrosis of the vertebral body. Spine (Phila Pa 1976) 2003;28:1588-92.

7. Deramond H, Depriester C, Galibert P, Le Gars D. Percutaneous vertebroplasty with polymethylmethacrylate: technique, indications, and results. Radiol Clin North Am 1998;36:533-46.

8. Kim DY, Lee SH, Jang JS, Chung SK, Lee HY. Intravertebral vacuum phenomenon in osteoporotic compression fracture: report of 67 cases with quantitative evaluation of intravertebral instability. J Neurosurg 2004;100(1 Suppl Spine):24-31.

9. Nakamae T, Fujimoto Y, Yamada K, Hashimoto T, Olmarker K. Efficacy of percutaneous vertebroplasty in the treatment of osteoporotic vertebral compression fractures with intravertebral cleft. Open Orthop J 2015;9:107-13.

10. Nakamae T, Fujimoto Y, Yamada K, Takata H, Shimbo T, Tsuchida Y. Percutaneous vertebroplasty for osteoporotic vertebral compression fracture with intravertebral cleft associated with delayed neurologic deficit. Eur Spine J 2013;22:1624-32.

11. Levine SA, Perin LA, Hayes D, Hayes WS. An evidence-based evaluation of percutaneous vertebroplasty. Manag Care 2000;9:56-60,63.

12. Ha KY, Kim KW, Kim YH, Oh IS, Park SW. Revision surgery after vertebroplasty or kyphoplasty. Clin Orthop Surg 2010;2:203-8.

13. Alvarez L, Perez-Higueras A, Granizo JJ, de Miguel I, Quinones D, Rossi RE. Predictors of outcomes of percutaneous vertebroplasty for osteoporotic vertebral fractures. Spine (Phila Pa 1976) 2005;30:87-92.

14. Ha KY, Kim YH, Chang DG, Son IN, Kim KW, Kim SE. Causes of late revision surgery after bone cement augmentation in osteoporotic vertebral compression fractures. Asian Spine J 2013;7:294-300.

15. Hirakawa M, Kobayashi N, Ishiyama M, et al. Radiological findings as favorable predictors of pain relief in patients with osteoporotic compression fractures after percutaneous vertebroplasty: a retrospective study of 156 cases. Jpn J Radiol 2012;30:407-14.

16. Maynard AS, Jensen ME, Schweickert PA, Marx WF, Short JG, Kallmes DF. Value of bone scan imaging in predicting pain relief from percutaneous vertebroplasty in osteoporotic vertebral fractures. AJNR Am J Neuroradiol 2000;21:1807-12.

17. Kaufmann TJ, Jensen ME, Schweickert PA, Marx WF, Kallmes DF. Age of fracture and clinical outcomes of percutaneous vertebroplasty. AJNR Am J Neuroradiol 2001;22:1860-3.

18. Fairbank JC, Pynsent PB. The Oswestry Disability Index. Spine (Phila Pa 1976) 2000;25:2940-52.

19. Fairbank JC, Couper J, Davies JB, O’Brien JP. The Oswestry low back pain disability questionnaire. Physiotherapy 1980;66:271-3.

20. Chen LH, Hsieh MK, Liao JC, et al. Repeated percutaneous vertebroplasty for refracture of cemented vertebrae. Arch Orthop Trauma Surg 2011;131:92733.

21. Lin WC, Lee YC, Lee CH, et al. Refractures in cemented vertebrae after percutaneous vertebroplasty: a retrospective analysis. Eur Spine J 2008;17:592-9.

22. Genever RW, Downes TW, Medcalf P. Fracture rates in Parkinson's disease compared with age- and gender-matched controls: a retrospective cohort study. Age Ageing 2005;34:21-4.

23. Babat LB, McLain RF, Bingaman W, Kalfas I, Young P, Rufo-Smith C. Spinal surgery in patients with Parkinson's disease: construct failure and progressive deformity. Spine (Phila Pa 1976) 2004;29:2006-12.

24. Koller H, Acosta F, Zenner J, et al. Spinal surgery in patients with Parkinson's disease: experiences with the challenges posed by sagittal imbalance and the Parkinson's spine. Eur Spine J 2010;19:1785-94.

25. Moon SH, Lee HM, Chun HJ, et al. Surgical outcome of lumbar fusion surgery in patients with Parkinson disease. J Spinal Disord Tech 2012;25:351-5.

26. Kawaguchi Y, Nakano M, Seki S, et al. Surgical treatment of lumbar vertebral collapse in a patient with Parkinson's disease: a case report. Eur J Orthop Surg Traumatol 2013;23 Suppl 2:S165-70.

27. Aoki Y, Nakajima A, Sakakibara R, Ohtori S, Takahashi K, Nakagawa K. Pathologic thoracic spine fracture in presence of Parkinson's disease and diffuse ankylosis: successful management of a challenging condition. BMC Musculoskelet Disord 2013;14:61.

28. Nakashima H, Yukawa Y, Ito K, Horie Y, Machino 
M, Kato F. Combined posteroanterior surgery for osteoporotic delayed vertebral fracture and neural deficit in patients with Parkinson's disease. Orthopedics 2009;32. https://doi.org/10.3928/0147744720090818-21.
29. Denis F. Spinal instability as defined by the threecolumn spine concept in acute spinal trauma. Clin Orthop Relat Res 1984;(189):65-76.

30. Seo MR, Park SY, Park JS, Jin W, Ryu KN. Spinous process fractures in osteoporotic thoracolumbar vertebral fractures. Br J Radiol 2011;84:1046-9. 